[14] Marker, C. P., 1926. Communication personnelle.

[15] Marker C. P. Carte de classement de beurre par points, Form. D. 16, 30005-14, Alberta, Dept. of Agr.

[16] Sтовсн V. 1890 (Trad.), 18 Rapport du Laboratoire de Recherches agricoles. Laboratoire de Recherches Agricoles. Copenhague.

[17] Jensen, C. O., 1891 (Trans.) 32e Rapport du Laboratoire de Recherches $d u$ Collège Royal Vétérinaire et Agricole. Copenhague. p. 24 et p. 59.

[18] Todd, Alex. et SAdLer, Wilfrid, 1911, Le fromage de Kingston, Jour. Bd of Agr. XVIII, 3, pp. 193-203, London.

[19] KenLy, C. D., 1924, La bactériologie du fromage de Kingston. Trans Roy. Socy. Can. 3e Série, XVIII, pp. 51-59, Ottawa.

[20] SADLER, Wilfrid, 1926, La flore bactérienne du fromage de Kingston montrée par les micro-photographies. Trans. Roy. Socy. Can. et Le Lait 1927, $\mathrm{n}^{\circ} 1$.

\title{
LES INDICATEURS ET L'ACIDITÉ TOTALE ET RÉELLE DU LAIT
}

\author{
par $\mathrm{D}^{\mathrm{r}}$ Alb. J.-J. VANDEVELDE
}

Professeur à l'Institut Supérieur Agronomique et à l'Université de Gand, Directeur de i'Institut Supérieur des Fermentations.

La détermination de l'indice de la concentration en ions hydrogène, que Sörensen a désigné par le symbole $\mathrm{pH}$, et que Clark a proposé de nommer degré Sörensen (1), se fait par voie électrique et par voie colorimétrique. La méthode électrique est sans doute la plus exacte, quand elle est adroitement et souvent appliquée; la méthode colorimétrique est peut-être moins précise, mais elle est d'exécution rapide et facile, et elle offre l'avantage d'être à la portée de chacun.

La méthode colorimétrique n'est cependant bien applicable qu'à des liquides transparents : les liquides troubles, les solutions colloïdales ne peuvent pas toujours être employés. Le lait est dans ce cas; les colorants de Sörensen, ceux de ClaRK et LuBBS donnent des résultats très irréguliers; ceux de MicHAfLIs ne sauraient être employés. Il est préférable de transformer, pour l'emploi des indicateurs, les liquides colloïdaux en solutions limpides.

Schultz et Chaudler (2) ont proposé de déterminer le degré Sörensen en dialysant le lait à l'aide d'un sac de collodion; ils emploient un volume de lait et comme récepteur 2 volumes d'eau distillée très pure, puis ils soumettent le dialysat à l'examen colorimétrique. Ils ont trouvé ainsi $\mathrm{pH}=6.53$ en moyenne, entre 6,7 et 6,4 pour le lait de chèvre, $\mathrm{pH}=7,22$ pour le lait humain.

(1) The determination of hydrogen ions; Baltimore, 1922, p. 13.

(2) The acidity of goats milk in terms of hydrogen ion concentration, with cotaparisons to that of eow's and human milk; Journ. biol. Ohem., t. 46, pp. 129-131. 
HrRsch (3) recommande pour le titrage des ampholytes l'emploi du fer colloïdal préconisé par SoxHLET et Henkel : $50 \mathrm{cc}$. de lait sont traités par 38 ce. Liq. ferri oxydati dialysat., et après 15 minutes de repos, il suffit de filtrer pour obtenir un sérum limpide.

Quand il s'agit de lait acide, l'obtention d'un sérum limpide n'offre aucune difficulté; la coagulation d'une partie des protéines permet la séparation de la substance qui la rend opaque, et on peut ajouter, comme l'indique HoLwerda (4) de la terre à infusoires bien neutre pour obtenir un liquide parfaitement limpide sans que l'indice $\mathrm{pH}$ soit modifié; dans ce eas, le rouge de méthyle donne les mêmes résultats que la méthode électrométrique.

Dans son importante étude sur l'influence de la réaction du milieu sur les phénomènes de la coagulation du lait, W. VAN DAM (5) donne quelques indications intéressant les rapports entre les indicateurs et l'acidimétrie du lait. Il signale notamment qu'avec l'acide rosolique on arrive à une acidité totale ou acidité titrée (getitreerde zuurgraad) plus faible qu'avec la phénolphtaléine : l'acide rosolique donne ainsi le virage rouge quand la teneur en ions $\mathrm{OH}$ du lait est encore trop faible pour virer avec la phénolphtaléine au rouge; le papier de tournesol donne aussi des valeurs plus faibles que la phénolphtaléine; le rouge de méthyle donne un virage rouge quand l'orange de méthyle en est encore à la coloration jaune.

L'alizarine est employée par MonRes pour établir l'état de fraîcheur du lait (6); dans la gamme des couleurs, le lait normal est rouge violet, le lait alcalin est plus violet, le lait devenant acide est rouge brun, et pour une acidité maximale, la coloration est jaune. Avec le lait de -vaches atteintes de mammite, et dans lequel il y a souvent un excédent d'ions $\mathrm{OH}$, l'acide rosolique donne une coloration rouge.

BAKER et VAN SLYKKE (7) ont obtenu avec le pourpre de bromocresol une eoloration bleu-gris pour le lait normal, une coloration bleu foncé avec les laits alcalins, par exemple ceux des vaches atteintes de mammite. Kolthoff (8) utilise le rouge de phénol pour distinguer, à l'aide d'une échelle de teintes, l'acidité réelle du lait : pour une acidité réelle faible la teinte est vers le rouge, pour une acidité réelle élevée, la teinte est vers le jaune. La méthode de Cooledge (9) fait usage de 0,1 cc. de lait que l'on introduit dans du bouillon stérile additionné de bleu de bromothymol, et on détermine la teinte d'heure en heure, dans la culture maintenue à $37^{\circ} \mathrm{C}$.

\footnotetext{
(3) Biochem-Zeitschr., 1924, 147, p. 423.

(4) Verst. landb. Onderz. Rykslandbouwproefst., 1925, 30, pp. 220-223.

(5) Opstellen over moderne Zuivelchemie, 1922, p. 25.

(6) Zeitschr. Unters. Nahrungsm, 1911, 22, p. 459.

(7) Journ. biol. Chem., 1919, 40, p. 335.

(8) Handelingen Genootschap Melkkunde, 1920.

(9) Ayric. Bxp. Station Michigan, Bull. 52, nov. 1921.
} 
VAN DAM ne se montre cependant pas enthousiaste au sujet des résultats colorimétriques obtenus, et je partage cette opinion. Il n'est pas étonnant en effet que les indieateurs donnent des acidités totales très divergentes, et que les divers dosages faits aveo le même indicateur ne soient pas toujours concordants. Le lait n'est pas seulement un mélange complexe dans lequel des ions $\mathrm{H}$ ou des ions $\mathrm{OH}$ peuvent être prédominants, mais la présence des phosphateset des protéines complique singulièrement la réaction du milieu, sans compter avec la possibilité d'une combinaison des colorants avec les protéines. De VARDA (10) fait également des réserves très sérieuses au sujet de l'essai colorimétrique avec l'alizarine.

KorтhоғF (11) indique pour le lait eru un degré Sörensen 6,6 à 7,6 ; il recommande comme indicateur le rouge de phenol que le lait normal colore en une teinte intermédiaire et dont le degré Sörensen peut alors être évalué à l'aide d'une échelle de coloration. La teinte peut être plus facilement appréciée quand on ajoute au lait un peu d'oxalate de potassium, mais la réaction devient alors plus fortement alealine. BAKER et VAN SLYKE (12) donnent la préférence au pourpre de bromocrésol dont une goutte de solution saturée donne avec 3 ec. de lait une coloration gris bleu; cette coloration devient plus claire avec les acides et par le chauffage au delà du point de pasteurisation; elle devient au contraire plus foncée quand le lait a été additionné d'eau ou d'un sel à réaction alealine, ou quand le lait provient de vaches atteintes de mastite.

Par électrométrie, FABER et HaDdEN (13) ont trouvé pour le lait de vache un $\mathrm{pH}$ moyen $=6,89$, pour le colostrum 7,74; l'addition d'eau modifie le $\mathrm{pH}$ : on trouve 6,96 pour un mélange de 2 volumes de lait et 1 volume d'eau, 7,09 pour un mélange de 1 volume de lait et 2 volumes d'eau. Le lait ensemencé par Bacillus acidophilus accuse 6,48 après 12 heures, 6,02 après 18 heures, 5,52 après 24 heures. Par électrométrie également, DunCombe (14) a obtenu 6,17 à $17^{\circ} \mathrm{C}$. et 5.89 à $50^{\circ} \mathrm{C}$.; toutefois cet auteur doit avoir opéré súr du lait en voie d'acidification, puisque dans son mémoire il rapporte que la neu. tralisation, avec la phénolphtaleine comme indicateur, a exigé pour $100 \mathrm{cc}$ de lait $31.7 \mathrm{cc}$. de solution $\frac{\mathrm{N}}{10} \mathrm{OH} \cdot$ à $21^{\circ} \mathrm{C}$ et $42,3 \mathrm{ce}$. de la même solution à $72^{\circ} \mathrm{C}$.

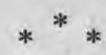

J'ai déterminé la réaction du lait centrifugé stérilisé, avant et après

(10) Milchw. Zentralbl., 1914, 43, p. 154.

(11) Der Gebrauch von Farbenindicatoren, Berlin 1921, p. 108.

(12) Journ. Biol. Chem., 1919, 40, p. 357.

(13) Med. Clin. N. Amor., 1922, 6, pp. 245-261.

(14) Le Lait, Lyon, 1926, p. 117. 
infection, soit par une trace de lait cru contenant done une grande variété de microbes, mais essentiellement des bactéries lactiques, soit par diverses souches de Bacterium Coli. Les résultats sont exprimées en ce. 0 , In pour $10 \mathrm{cc}$. de lait, avec la prédominance réactionnelle vis-à-vis des indicateurs, telle que le lait l'a montrée; cette prédominance réactionnelle est traduite dans mes tableaux par $\mathrm{H}$ et par $\mathrm{OH}$.

J'ai obtenu un sérum très limpide en traitant $100 \mathrm{cc}$. de lait par 1 cc. de solution $\frac{n}{2}$ de chlorure de baryum $\left(122,2\right.$ gr. $\mathrm{Ba} \mathrm{Cl}_{2} \cdot 2 \mathrm{H}_{2} \mathrm{O}$ au litre) et 99 cc. d'éthanol neutre à $94 \mathrm{~V} \%$ Le filtrat a servi à la détermination du degré SöRENSEN par la méthode colorimétrique de Mrchateris ; le nitrophénol a donné ainsi des résultats très constants, plus réguliers que les colorants de CLARK dont la teinte était difficile à comparer avec les tests. La colorimétrie du nitrophénol a comme termes de comparaison les échelles de WINDISCH au chromate et au bichromate, ainsi que les solutions de phosphate de SöRENSEN. Le sérum obtenu a été aussi analysé volumétriquement en présence de divers indicateurs, eomme pour le lait, et les résultats obtenus, multipliés par 2, correspondent ainsi à 10 ce. de lait initial; ce sont ces valeurs ainsi calculées, et donc comparables à celles du lait lui-même, qui sont données dans les tableaux, avec l'indication de la prédominance réactionnelle pour chacun des indicateurs.

J'ai employé les indicateurs suivants:

phénolphtaleine $(\mathrm{Php})$, incolore à rouge, $\mathrm{pH} 8,3$ à 10 comme zone de virage.

rouge neutre $(\mathrm{Rne})$, rouge à orange, $\mathrm{pH} 6,8$ à 8 .

thymolphtaléine (Thp), incolore à bleu, $\mathrm{pH} 9,5$ à 10,5

naphtolphtaléine (Nap), rose à bleu, $\mathrm{pH} 7,3$ à 8,7

tournesol (to), rouge à bleu, $\mathrm{pH} 5,0$ à 8,0

rouge de méthyle (Rme), rose à jaune, $\mathrm{pH} 4,5$ à 6,0

lacmoïde (Lacm), rouge à bleu, $\mathrm{pH} 4,4$ à 6,4

alizarine (aliz.), jaune à rose violet, $\mathrm{pH} 10,1$ à 12,1

orange de méthyle (Orme), rose à orange, $\mathrm{pH} 3,1$ à 4,4

acide rosolique (Ac ros), jaune à rose, $\mathrm{pH} 6,9$ à 8,0

rouge Congo (Reo), bleu à rouge, $\mathrm{pH} 3,0$ à 5,2

Le degré SöRENSEN obtenu pour le sérum correspond très bien aux données signalées pour le lait chauffé. En effet W. Van Dam (15) indique comme acidité réelle $0,19 \times 10^{-6}$ pour le lait cru et $0,29 \times$ $10^{-6}$ pour le lait chauffé, ce qui correspond à $1,9 \times 10^{-7}=10^{0,28} \times$ $10^{-7}=10^{-6,72}=\mathrm{pH} 6,72$ pour le lait crû, - et à $2,9 \times 10^{-7}=10^{0,47}$ $\times 10^{-7}=10^{-6,53}=\mathrm{pH} 6,53$ pour le lait chauffé. Or le sérum que j'ai

(15) Opstellen over moderne Zuivelchemie, 1922, p. 21. 
obtenu a donné par la méthode eolorimétrique de Mrchazus pH 6,3 à 6,5 .

Dans une première série d'essais, $\mathrm{j}$ 'ai fait usage des 11 indicateurs signalés plus haut pour déterminer l'acidité du lait avant et après infection par une trace de lait cru. Les résultats obtenus pour le lait et pour le sérum préparé d'après ma méthode sont très variables, ainsi que le montre l'inspection du tableau I.

La phénolphtaléine, le rouge neutre, la thymolphtaléine et la naphtolphtaléine donnent une prédominance à l'ion $\mathrm{H}^{+}$, les autres indicateurs à l'ion $\mathrm{OH}-$; la php., le Rne et la nap. donnent des résultats assez concordants, la thp. conduit à des valeurs sensiblement doubles; le lacm., l'aliz, le orme, l'acros donnent des valeurs très élevées, et avec le Reo, le virage ne se produit pas de manière appréciable. Quand le lait s'acidifie, le to et le Rme accusent des ions $\mathrm{H}^{+}$, le lacm, l'aliz, le orme, l'acros et le Reo maintiennent la prédominance alcaline.

Quant au sérum, les valeurs observées sont toujours inférieures à celles obtenues pour le lait lui-même; il manifeste en général une prédominance d'ions $H$, excepté en présence de lacm, alzi, orme, Reo qui maintiennent dans les laits acidifiés une prédominance pour les ions $\mathrm{OH}$; pour ce dernier indicateur, il faut une grande quantité de solution $\mathrm{n} / 10$ acide pour arriver à un virage appréciable.

KoLthoFf (16) signale que pour le rouge de méthyle dont la zone de virage est comprise entre $\mathrm{pH} 4.5$ et $\mathrm{pH} 6.0$, le maximum d'intensité colorante se trouve pour $\mathrm{pH}$ compris entre 3 et 4 ; quand le virage se fait pour un $\mathrm{pH}$ entre 4 et 6 , la substance se comporte comme une base. J'observe le même fait à propos du lait dont le degré SöRENSEN est voisin de 6.5, mais je ne l'observe plus quand il s'agit du sérum préparé selon ma méthode. Le méthylorange qui est un indicateur basique montre dans le lait une prédominance constante pour les ions $\mathrm{OH}$ (17); la solution de biphtalate doit être remplacée, pour la détermination colorimétrique, par la solution de citrate recommandée par Sörensen.

$$
\text { ** * }
$$

Pour l'infection du lait par Bacterium Coli, je me suis servi de cultures pures de diverses origines, conservées sur bouillon gelosé et rajeunies par deux repiquages en bouillon peptoné au moment de l'emploi. Les souches B4 et B5 proviennent de la School of Hygiene and Public Health de l'Université John Hopkins à Baltimore; les souches F1, F2, F3, F5, F6, F7 sont originaires du Stanford Hospital de San Francisco, service du doeteur Wm. Opfals. Les souches de Baltimore et de San Francisco m'ont été rapportées par le docteur Jean VANDE-

(16) Rec, trav, chim. Pays-Bas, 1925, 44, p. 75.

(17) I. M. Kоцтноут, Rec. trav, chim. Pays-Bas, 1925,44 p. 66 ; 1926, 45, p. 433. 


\section{TABLEAU I}

20 ce. sérum, soit 10 ce. lait $\} \mathrm{n} / 10$

Lait 1014 stérilisé

Lait 1014 + traces lait cru, $3 \mathrm{j}$. . . . .

Id., sérum . . . . . . . . . . .

Lait 1030, stérilisé $\ldots \ldots \ldots \ldots \ldots \ldots$

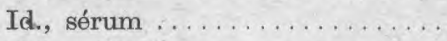

Lait 1102, stérilisé . . . . . . . . . .

Id., serum . . . . . . . . . . .

Lait 1102 + traces lait cru, 2 j. . . . .

Id., serum $\ldots \ldots \ldots \ldots \ldots \ldots$

Lait 1107, stérilisé

Id., serum . . . . . . . . . . .

Lait 1107 + traces lait eru, 2 j. . . . . . Id., serum ...............

Lait 1218, stérilisé $\ldots \ldots \ldots \ldots \ldots \ldots \ldots$

Id., serum . . . . . . . . . .

Lait 1218 + traces lait eru, $3 \mathrm{j} . \ldots \ldots$. Id., serum

\begin{tabular}{|c|c|c|c|c|c|c|}
\hline Plop & Rne & Thp & Nap & To & Rme & Lacm \\
\hline $\mathrm{H}$ & H & H & H & & & $\mathrm{OH}$ \\
\hline 1,5 & 1,1 & 3,6 & 1,4 & $2,0^{\mathrm{OH}}$ & $1,3^{\mathrm{OH}}$ & 9,0 \\
\hline 4,7 & 5,1 & 6,0 & 5,4 & $2,0^{\mathrm{H}}$ & $1,0^{\mathrm{H}}$ & 9,0 . \\
\hline 4,0 & - & - & 3,0 & $1,8^{\mathrm{H}}$ & $0,8^{\mathrm{H}}$ & - \\
\hline 1,5 & 1,3 & 3,8 & 1,2 & $2,2^{\mathrm{OH}}$ & 1,9 ОН & 8,0 \\
\hline 1,4 & 0,6 & 1,6 & 0,4 & $1,0^{\mathrm{OH}}$ & $0,2^{\mathrm{H}}$ & 2,0 \\
\hline 1,9 & 1,7 & 3,6 & 1,5 & $1,8^{\mathrm{OH}}$ & $1,3^{\mathrm{OH}}$ & 8,0 \\
\hline 1,4 & 0,4 & 1,4 & 0,8 & $0,6^{\mathrm{OH}}$ & $0,2^{\mathrm{H}}$ & 2,0 \\
\hline 5,4 & 4,8 & 7,5 & 4,8 & $2,0^{\mathrm{H}}$ & $1,0^{\mathrm{H}}$ & 9,0 \\
\hline 3,6 & 2,2 & 3,8 & 2,4 & $1,6^{\mathrm{H}}$ & $0,8^{\mathrm{H}}$ & 2,0 \\
\hline 1,7 & 1,6 & 3,7 & 1,5 & $1,4^{\mathrm{OH}}$ & $1,1^{\mathrm{OH}}$ & 9,0 \\
\hline 1,0 & 0,4 & 1,2 & 0,4 & $0,4^{\mathrm{OH}}$ & $0,2^{\mathrm{H}}$ & 2,0 \\
\hline 3,0 & 2,6 & 4,8 & 2,5 & $1,5^{\mathrm{H}}$ & $0,8^{\mathrm{H}}$ & 7,0 \\
\hline 1,8 & 1,0 & 2,0 & 1,6 & $0,6^{\mathrm{H}}$ & $0,6^{\mathrm{H}}$ & 1,8 \\
\hline 1,8 & 1,4 & 3,5 & 1,3 & $2,1^{\mathrm{OH}}$ & $1,5^{\mathrm{OH}}$ & 8,2 \\
\hline 1,2 & 0,6 & 1,0 & 0,6 & $0,8^{\mathrm{OH}}$ & $0,2^{\mathrm{H}}$ & 1,0 \\
\hline 3,1 & 2,0 & 4,6 & 2,1 & $0,9^{\mathrm{H}}$ & $0,8^{\mathrm{OH}}$ & 8,4 \\
\hline 1,4 & 1,2 & 1,6 & 1,0 & $0,6^{\mathrm{H}}$ & $0,4^{\mathrm{H}}$ & 1,2 \\
\hline
\end{tabular}

$\begin{array}{rcc}\text { Orme } & \text { Ac ros } & \text { Reo } \\ & - & - \\ \text { OH } & & \text { он } \\ 9,9 & 8,0^{\mathrm{OH}} & \infty \\ 8,9 & 10,0^{\mathrm{OH}} & 20,0 \\ 1,6 & - & 16,0 \\ 10,0 & 8,0^{\mathrm{OH}} & \infty \\ 2,4 & 0,2^{\mathrm{H}} & 6,0 \\ 11,0 & 7,0^{\mathrm{H}} & \infty \\ 2,2 & 0,2^{\mathrm{H}} & 8,0 \\ 9,0 & 12,0^{\mathrm{OH}} & \infty \\ 2,0 & 0,8^{\mathrm{H}} & 16,0 \\ 10,0 & 8,0^{\mathrm{OH}} & \infty \\ 2,0 & 0,2^{\mathrm{H}} & 12,0 \\ 10,0 & 13,0^{\mathrm{OH}} & \infty \\ 3,2 & 0,8^{\mathrm{H}} & 1,6 \\ 10,3 & 8,2^{\mathrm{OH}} & \infty \\ 1,8 & 0,2^{\mathrm{H}} & 10,0 \\ 10,1 & 11,0^{\mathrm{OH}} & \infty \\ 2,2 & 0,4^{\mathrm{H}} & 22,0\end{array}$


VELDE, chargé de cours à l'Université de Gand. Enfin j'ai employé une souche G1 provenant directement de l'intestin humain normal, et une souche WI extraite d'une eau de grande pureté chimique. Toutes ces souches produisent de l'indol; les souches B5,F1,F2,F5 et F7 coagulent rapidement le lait, les autres ne provoquent guère de coagulation et produisent d'ailleurs moins d'acide que les premières, ainsi que le montrent les résultats de l'analyse volumétrique.

Les résultats obtenus avec la php, le Rne, la thp, la nap, le Rme et I'aliz. confirment les résultats du tableau I; l'acidité totale du sérum varie de la même manière que celle du lait. L'acidité réelle $\mathrm{pH} 6,5$ pour le lait frais s'élève à $\mathrm{pH} 5.1$ à 5.7 et suit les variations de l'acidité totale. On sait que Bact. Coli présente des races productrices d'acide, et des races peu acidifiantes ; parmi les races employées, j'ai constaté que l'une des souches de San Francisco F3, d'origine pathogène, donne des résultats presque identiques à ceux des deux souches que j'ai isolées moimême, l'une d'une eau de distribution Wl et l'autre d'un intestin non malade. Le tableau II contient l'aperçu des résultats obtenus pour les dix souches, le tableau III pour l'une d'entre elles, F1, qui est très active et qui a été mise en culture à 3 températures différentes $9^{\circ}, 16^{\circ}$ et $37^{\circ} \mathrm{C}$; le degré SöRENSEN qui pour le lait stérilisé était 6,3-6,4 a atteint la valeur maximale de $5,7,5,3$ et 5,1 , respectivement pour les trois températures.

Enfin j'ai comparé pour la php., la.thp., le Rme et l'aliz. l'action de 3 souches américaines actives F1, F2, B4 et des 2 souches W1 et G1 à la.température ordinaire, pendant une période de 19 jours. Les résultats qui confirment ceux que j'ai obtenus plus haut, 'sont réunis dans le tableau IV; on remarquera la constance des valeurs obtenues avec l'alizarine.

$$
\text { ** } *
$$

En solution aqueuse, l'acide lactique présente à la concentration $\frac{N}{10}$ un degré Sörensen 2,43 , avec une concentration en ions $\mathrm{H}+$ de $3,7 \times$ 10-3. Le lait est un milieu complexe dans lequel l'action de l'acide lactique ne se manifeste que partiellement; c'est ce qui explique les résultats très variables obtenus avec les différents indicateurs. Si la détermination de l'acidité réelle peut nous donner des indications exactes, la détermination de l'acidité totale ou titrée du lait est conventionnelle; on a l'habitude d'employer la phénolphtaléine comme indicateur, mais il ne faut pas perdre de vue que la valeur obtenue est conventionnelle et non conforme à la réalité. Le rouge neutre dont la zone de virage ( $\mathrm{pH} 6.8$ à 8.0 ) s'écarte de celle de la phénolphtaléine ( $\mathrm{pH} 8.3$ à 10.0) donne des valeurs qui se rapprochent le mieux de l'acidité titrée avec cette phénolphtaléine; la thymolphtaléine dont la zone de virage ( $\mathrm{pH} 9,5$ à 10,5) se rapproche de celle de la phénolphtaléine donne 
TABLEAU II

$\left.\begin{array}{c}10 \mathrm{cc} \text {. lait } \\ 20 \mathrm{cc} . \text { sérum, sort } 10 \mathrm{cc} \text {. lait }\end{array}\right\} \mathrm{n} / 10$

Lait stérile, Bm Coli O

Id., sérum

Bm Coli B4

B5
F1
F2
F3
F5
F6
F7
G1
W1

B5

F2

F6

F7

W1
Lait $+\mathrm{Bm}$ coli, $3 \mathrm{j} .16^{\circ} \mathrm{C}$

\begin{tabular}{|c|c|c|c|c|c|}
\hline Php & Rne & Thp & Nap & Rine & Aliz \\
\hline- & - & - & - & & - \\
\hline $\mathrm{H}$ & H & H & H & & $\mathrm{OH}$ \\
\hline 1,9 & 2,0 & 4,0 & 1,8 & $1,0^{\circ \dot{H}}$ & 8,0 \\
\hline- & - & - & - & - & - \\
\hline 4,9 & 4,3 & 7,5 & 4,7 & $1,7^{\mathrm{H}}$ & 8,5 \\
\hline 6,0 & 5,8 & 8,2 & 6,0 & $2,4^{\mathrm{H}}$ & 8,0 \\
\hline 6,4 & 6,3 & 8,6 & 6,2 & $2,6^{\mathrm{H}}$ & 7,8 \\
\hline 6,5 & 6,6 & 8,1 & 6,2 & $2,3^{\mathrm{H}}$ & 7,3 \\
\hline 2,5 & 2,4 & 4,3 & 2,4 & $0,4^{\mathrm{OH}}$ & 8,5 \\
\hline 5,3 & 5,0 & 7,0 & 5,4 & $1,8^{\mathrm{H}}$ & 8,0 \\
\hline 4,3 & 4,3 & 6,8 & 4,2 & $0,7^{\mathrm{H}}$ & 8,1 \\
\hline 6,3 & 6,0 & 8,4 & $\tilde{\delta}, 9$ & $2,0^{\mathrm{H}}$ & 7,9 \\
\hline 2,5 & 2,8 & 5,6 & 3,1 & $0,5^{\mathrm{OH}}$ & 7,8 \\
\hline 3,3 & 3,2 & 5,2 & 3,2 & $0,2^{\mathrm{OH}}$ & 7,8 \\
\hline
\end{tabular}

Lait $+\mathrm{Bm}$ coli, $16^{\circ} \mathrm{C}$, sérum

\begin{tabular}{|c|c|c|c|c|c|c|}
\hline Php & Rne & Thp & Nap & Rme & Aliz & $\overline{\mathrm{pH}}$ \\
\hline- & - & - & - & - & - & - \\
\hline $\mathrm{H}$ & H & $\mathrm{H}$ & H & & & \\
\hline$\ldots$ & 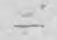 & - & - & - & - & T \\
\hline 1,8 & 1,0 & 2,0 & 1,4 & $0,2^{\mathrm{OH}}$ & $1,2^{\mathrm{OH}}$ & 6,5 \\
\hline 3,4 & 3,8 & 3,4 & 3,4 & $2,0^{\mathrm{H}}$ & $1,6^{\mathrm{H}}$ & $\dot{5}, 7$ \\
\hline 4,4 & 4,2 & 5,0 & 4,4 & $2,2^{\mathrm{H}}$ & $1,2^{\mathrm{H}}$ & 5,3 \\
\hline 4,8 & 4,2 & 5,2 & 4,6 & $2,8^{\mathrm{H}}$ & $2,2^{\mathrm{H}}$ & 5,4 \\
\hline 5,0 & 4,4 & 5,2 & 5,0 & $3,4^{\mathrm{H}}$ & $2,2^{\mathrm{H}}$ & 5,1 \\
\hline 1,8 & 1,2 & 2,0 & 1,4 & $0,6^{\mathrm{H}}$ & $\theta, 6^{\mathrm{H}}$ & 5,7 \\
\hline 4,0 & 3,2 & 4,0 & 3,8 & $2,0^{\mathrm{H}}$ & $2,8^{\mathrm{H}}$ & 5,2 \\
\hline 2,8 & 2,6 & 3,4 & 2,8 & $1,4^{\mathrm{H}}$ & $1,8^{\mathrm{H}}$ & 5,5 \\
\hline 2,2 & 4,0 & 4,4 & 4,2 & $2,6^{\mathrm{H}}$ & $2,4^{\mathrm{H}}$ & 5,2 \\
\hline 2,0 & 1,4 & 2,2 & 2,0 & $0,6^{\mathrm{H}}$ & $1,0^{\mathrm{H}}$ & 5,7 \\
\hline 2,0 & 1,6 & 2,4 & 2,0 & $0,6^{\mathrm{H}}$ & $0,8^{\mathrm{H}}$ & 5,7 \\
\hline
\end{tabular}

\section{TABLEAU III}

\begin{tabular}{|c|c|c|c|c|c|}
\hline Php & Rne & Thp & Nap & Rme & Aliz \\
\hline- & 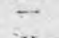 & - & - & & - \\
\hline
\end{tabular}

Lait stérilisé

$\begin{array}{llllllllll}\text { Lait }+\mathrm{Bm} \text { Coli F1, } & 9^{\circ} \mathrm{C} & 4 \mathrm{j} . & \ldots & 1,8 & 1,8 & 3,5 & 1,8 & 1,0^{\mathrm{OH}} & 8,0\end{array}$

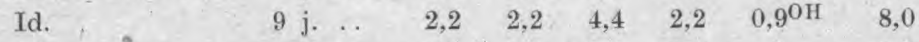

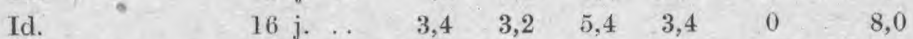

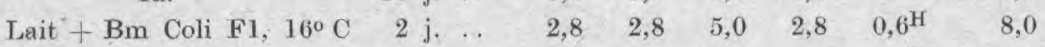

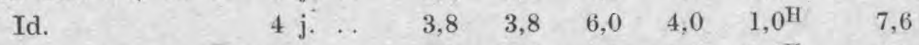

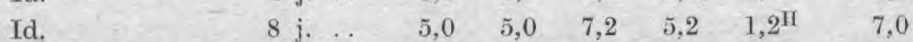

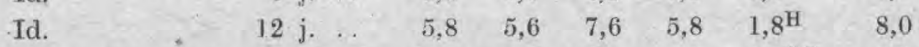

$\begin{array}{lllllllll}\text { Lait }+\mathrm{Bm} \text { Coli } \mathrm{F} 1,37^{\circ} \mathrm{C} & 2 \mathrm{j} . & \ldots & 6,1 & 6,1 & 8,1 & 6,0 & 1,0^{\mathrm{OH}} & 8,4\end{array}$

$\begin{array}{lllllllll}\text { Id. } & 5 \mathrm{j} . & \ldots & 6,4 & 6,5 & 8,5 & 6,2 & 2,5^{\mathrm{H}} & 6,9\end{array}$

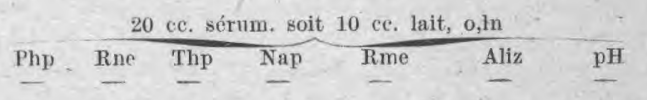

$\begin{array}{llll}\mathrm{H} & \mathrm{H} & \mathrm{H} & \mathrm{H}\end{array}$

$\begin{array}{lllllll}1,0 & 1,0 & 1,0 & 0,8 & 0,2^{\mathrm{OH}} & 0,8^{\mathrm{OH}} & 6,4\end{array}$

$\begin{array}{lllllll}1,0 & 0,8 & 1,4 & 1,0 & 0,2^{\mathrm{OH}} & 0,8 \mathrm{OH} & 6,4\end{array}$

$\begin{array}{lllllll}1,2 & 1,2 & 1,6 & 1,4 & 0,2^{\mathrm{OH}} & 0,8^{\mathrm{OH}} & 5,9\end{array}$

$\begin{array}{lllllll}2,0 & 2,0 & 2,4 & 2,0 & 0,8^{\mathrm{H}} & 1,2^{\mathrm{H}} & 5,7\end{array}$

$\begin{array}{lllllll}2,0 & 2,0 & 2,0 & 2,0 & 1,2^{\mathrm{H}} & 0,6^{\mathrm{H}} & 5,9\end{array}$

$\begin{array}{lllllll}3,2 & 3,2 & 3,6 & 3,6 & 2,0^{\mathrm{H}} & 0,8^{\mathrm{H}} & 5,6\end{array}$

$\begin{array}{lllllll}4,0 & 4,0 & 4,4 & 4,0 & 2,4^{\mathrm{H}} & 1,6^{\mathrm{H}} & 5,4\end{array}$

$\begin{array}{lllllll}4,4 & 4,4 & 4,4 & 4,4 & 3,6^{\mathrm{H}} & 2,0^{\mathrm{H}} & 5,3\end{array}$

$\begin{array}{lllllll}4,4 & 4,2 & 4,6 & 4,2 & 3,2^{\mathrm{H}} & 2,2^{\mathrm{H}} & 5,3\end{array}$

$\overline{ \pm}$ 
TABLEAU IV

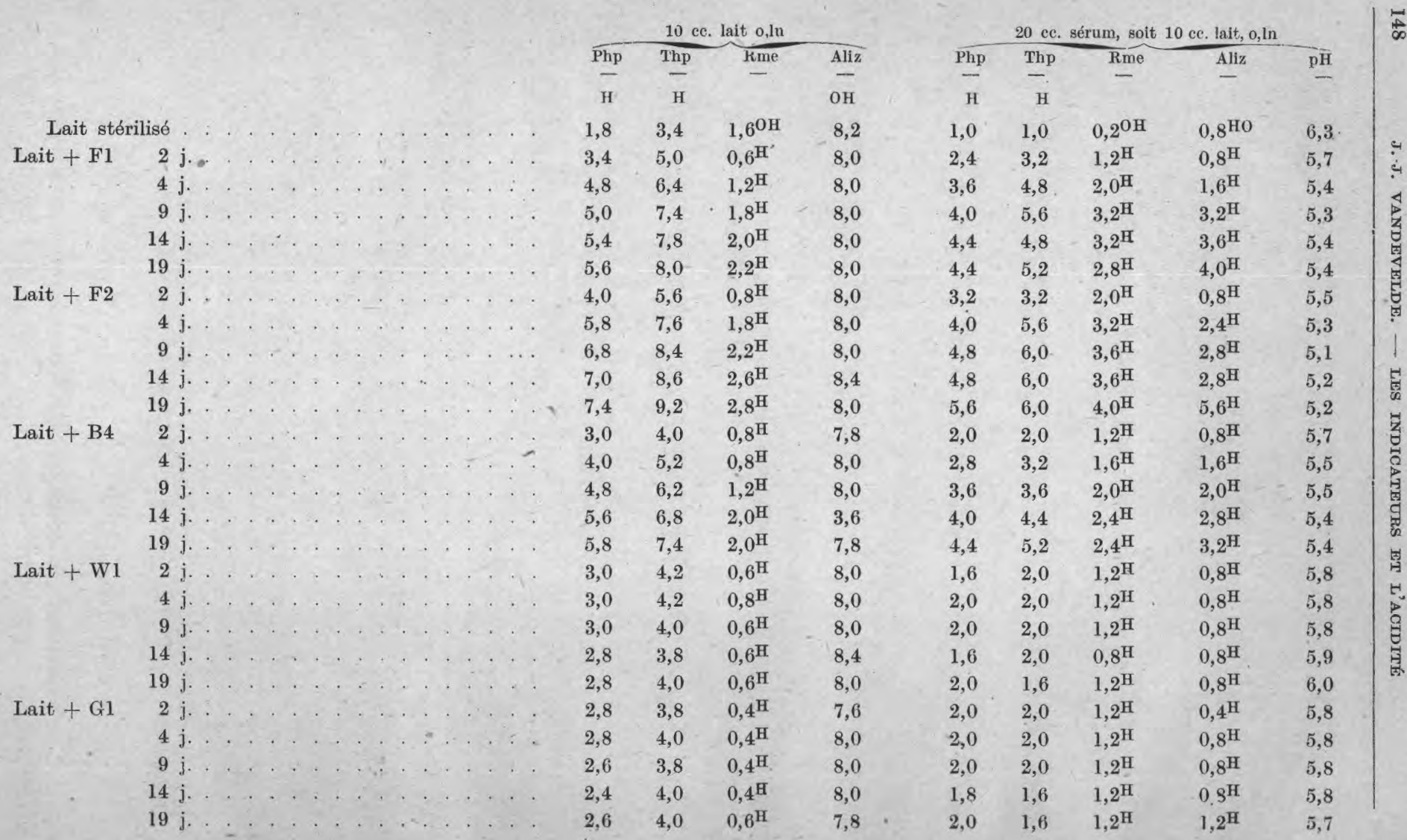


des valeurs variant du simple au double; le jaune d'alizarine $(\mathrm{pH} 10,1$ à 12,1) s'écarte totalement des valeurs fournies par la phénolphtaléine.

Je ne parviens pas à trouver, dans tous les chiffres fournis par mes nombreux essais, une concordance un peu précise entre le degré SörenSEN et l'acidité titrée ou totale du lait et du sérum obtenu par l'alcool en présence d'une trace de chlorure de baryum. Ceci montre bien que le degré d'acidité déterminé par simple volumétrie, en présence d'un indicateur comme la phénolphtaléine, doit être une valeur conventionnelle. Je me propose de poursuivre mes recherches à ce propos.

(Université de Gand, Laboratoire de chimie alimentaire.)

\title{
VALEUR DU LAIT EN CALORIES (1)
}

\author{
par O. R. OVERMAN et F. P. SANMANN \\ Section d'Economie Laitière \\ Université dillinors, Urbana, lllinois.
}

La connaissance de la "valeur (2) du lait en calories \#, c'est-à-dire du nombre de calories qui peut être produit par la combustion complète d'une quantité déterminée de lait, est à la fois intéressante et utile à ceux qui étudient la nutrition humaine et la nutrition animale.

Des formules ont été données pour le calcul de cette valeur. GAINES et DAVIDSON (a) travaillant sur des renseignements rassemblés par STOcking et BREW (b) ont donné la formule,

$$
\mathrm{E}=49,64(2,66+t)
$$

dans laquelle $\mathrm{E}$ est la chaleur de combustion de l'extrait sec du iait et $t$ le pourcentage de matière grasse. Cette formule qui donne la valeur énergétique par livre de lait est basée sur des moyennes d'analyses; elle fut employée pour apprécier la chaleur de combustion des laits de vaches.

Fredertisen (c) indique que le Directeur A. C. Andersen donne comme chaleur de combustion du lait: pourcentage de matière grasse $x$ $113.5+290$ ealories, par kilog.

L'étude actuelle a été commencée dans le but de déterminer les chaleurs de combustion, de laits de compositions différentes, de comparer les valeurs ainsi déterminées avee les chiffres correspondants établis d'après la composition de ces laits, et de s'assurer si des formules simples pouvaient être établies par des méthodes statistiques qui donneraient des chaleurs de combustion d'une exactitude suffisante pour les usages pratiques. Ces formules seront comparées avec les formules de Gatnes et Davidson, et d'Andersen.

(1) Une étude plus complète sur ce sujet paraitra sous forme de Bulletin à la Station d'Essais Agrieoles d'Illinois.

(2) Quand il est question de calorie, c'est toujours la grande calorie qui est envisagee. 